\title{
Close binary companions of the HAeBe stars LkH $\alpha$ 198, Elias 1, HK Ori and V380 Ori ${ }^{\star}$
}

\author{
K. W. Smith ${ }^{1}$, Y. Y. Balega ${ }^{2}$, W. J. Duschl ${ }^{3}$, K.-H. Hofmann ${ }^{1}$, R. Lachaume ${ }^{1}$, T. Preibisch ${ }^{1}$, \\ D. Schertl ${ }^{1}$, and G. Weigelt ${ }^{1}$ \\ ${ }^{1}$ Max-Planck-Institut für Radioastronomie (MPIfR), Auf dem Hügel 69, 53121 Bonn, Germany \\ e-mail: kester@mpifr-bonn.mpg.de \\ 2 Special Astrophysical Observatory, Nizhnij Arkhyz, Zelenchuk region, Karachai-Cherkesia 357147, Russia \\ 3 Institut für Theoretische Astrophysik, Tiergartenstrasse 15, 69121 Heidelberg, Germany
}

Received 21 April 2004 / Accepted 19 October 2004

\begin{abstract}
We present diffraction-limited bispectrum speckle interferometry observations of four well-known Herbig Ae/Be (HAeBe) stars, LkH $\alpha$ 198, Elias 1, HK Ori and V380 Ori. For two of these, LkH $\alpha 198$ and Elias 1, we present the first unambiguous detection of close companions. The plane of the orbit of the new $\mathrm{LkH} \alpha 198$ companion appears to be significantly inclined to the plane of the circumprimary disk, as inferred from the orientation of the outflow. We show that the Elias 1 companion may be a convective star, and suggest that it could therefore be the true origin of the X-ray emission from this object. In the cases of HK Ori and V380 Ori, we present new measurements of the relative positions of already-known companions, indicating orbital motion. For HK Ori, photometric measurements of the brightness of the individual components in four bands allowed us to decompose the system spectral energy distribution (SED) into the two separate component SEDs. The primary exhibits a strong infrared excess which suggests the presence of circumstellar material, whereas the companion can be modelled as a naked photosphere. The infrared excess of HK Ori A was found to contribute around two thirds of the total emission from this component, suggesting that accretion power contributes significantly to the flux. Submillimetre constraints mean that the circumstellar disk cannot be particularly massive, whilst the near-infrared data indicates a high accretion rate. Either the disk lifetime is very short, or the disk must be seen in an outburst phase.
\end{abstract}

Key words. stars: circumstellar matter - stars: formation - stars: binaries - ISM: Herbig-Haro objects

\section{Introduction}

Herbig Ae/Be stars (HAeBes) are young intermediate-mass (2-8 $M_{\odot}$ ) pre-main-sequence stars first defined by Herbig (1960). According to his definition, HAeBes should have emission lines in their spectra, an early spectral type and be associated with dark cloud material as an indication of their youth. Despite the similarities between HAeBes and the lower-mass $\mathrm{T}$ Tauri stars, questions such as the existence of circumstellar disks, the multiplicity and clustering of the objects, the origin of X-ray emission (Zinnecker \& Preibisch 1995), and the outflow activity are much less clarified in the case of HAeBes.

The frequency and properties of binary systems amongst all stars are of great importance in understanding the star formation process, since they can point to certain star formation scenarios and rule out or strongly constrain others.

* Based on observations performed with the $6 \mathrm{~m}$ telescope of the Special Astrophysical Observatory, Russia, the $2.2 \mathrm{~m}$ ESO/MPG telescope at La Silla, and with the NASA/ESA Hubble Space Telescope, obtained from the data archive at the Space Telescope Institute. STScI is operated by the association of Universities for Research in Astronomy, Inc. under the NASA contract NAS 5-26555.
The configuration of pre-main-sequence multiples is of special importance because the binary properties of the objects can be compared with their formation environment, for example whether in a $\mathrm{T}$ association or massive star forming region. There is also less scope for the system configuration to have been altered by stellar interactions or continuing accretion. The angular momentum of the binary can also be compared to that of circumstellar or circumbinary disks which may still exist at this stage. The coplanarity of the system orbit, the individual disks of the components and a possible circumbinary disk are all major constraints on viable formation mechanisms.

Numerous studies using a variety of techniques have established that the majority of low-mass young stars are members of binary systems (e.g. Ghez et al. 1993; Leinert et al. 1993; Simon et al. 1995). The T Tauri population in the nearby Taurus-Auriga T-association displays a factor of 2 higher binary fraction than that seen amongst main-sequence stars. At the high end of the stellar mass range, bispectrum speckle interferometry of the massive stars in the Orion nebula cluster revealed companions for 7 out of 13 target objects, which after correction for undetected systems was estimated to represent a binary frequency close to $100 \%$, with an average 
of $>1.5$ companions per primary (Preibisch et al. 1999). This is about three times the multiplicity of low-mass stars, and may suggest an alternative formation scenario for the high mass objects. Studies aimed specifically at intermediate mass stars have been rare. Li et al. (1994) carried out an infrared imaging study of 16 sources and reported companions for nine of them, although of these many were widely separated and are probably not bound. Leinert et al. (1997, hereafter LRH97) studied a larger sample of 31 stars using a speckle interferometry technique in the near-infrared, and found that $31 \%$ of the systems were multiple with separations between 50 and $1300 \mathrm{AU}$. This was estimated to represent an excess binarity among HAeBe stars compared to main-sequence $\mathrm{G}$ dwarfs of a factor of two. Pirzkal et al. (1997) used a speckle shift and add technique to search for companions around $39 \mathrm{HAeBe}$ stars. They detected 9 multiple systems, and estimated on statistical grounds that this implied a true binary frequency amongst HAeBes of at least $85 \%$. A survey for spectroscopic binaries amongst $\mathrm{HAeBe}$ stars by Corporon \& Lagrange (1999) found a binary frequency of $17 \%$. For short periods ( $<100$ days) the binary frequency was found to be $10 \%$, compared to the frequency amongst WTTS spectroscopic binaries of $11 \pm 4 \%$ (Mathieu 1992) or the frequency amongst main sequence solar-mass stars of $7 \pm 2 \%$ (Duquennoy \& Mayor 1991).

In this paper, we present bispectrum speckle interferometry at multiple epochs of four HAeBe systems. For two of these, $\mathrm{LkH} \alpha 198$ and Elias 1, our diffraction-limited images show unambiguously for the first time that they have close binary companions. For the known binary HK Ori, we separate the SEDs of the two companions and by considering the different colours show that the brighter object possesses a circumstellar disk which the secondary lacks. For V380 Ori, we are able to show relative motion compared to the position measured by LRH97.

\section{Observations and data reduction}

The optical observations of HK Ori were made with the $2.2 \mathrm{~m}$ ESO/MPG telescope on March 8, 1995. The speckle interferograms were recorded through interference filters with central wavelength/bandwidth of $550 \mathrm{~nm} / 60 \mathrm{~nm}$ and $656 \mathrm{~nm} / 60 \mathrm{~nm}$. The detector used for the visible observations was an image intensifier (gain 500 000) coupled optically to a fast CCD camera $\left(512^{2}\right.$ pixels/frame, frame rate 4 frames/s). All the other speckle interferograms were recorded with the SAO $6 \mathrm{~m}$ telescope in Russia between 1995 and 2003. The detector of our speckle camera at SAO was either a Rockwell HAWAII array (1998-2003; only one $512 \times 512$ Quadrant was used) or a $256 \times 256$ PICNIC array detector (1996 and 1997). The visible data sets consist of about 700 to 1400 speckle interferograms each with an exposure time of $50 \mathrm{msec}$. The infrared sets consist of between 400 and 3200 speckle interferograms each with an exposure time of between 150 and $250 \mathrm{msec}$.

The object power spectra were determined with the speckle interferometry method (Labeyrie 1970). Speckle interferograms of unresolved single stars were recorded just before and after the object and served as references to determine the speckle transfer function. Diffraction-limited images were reconstructed using the bispectrum speckle interferometry method (Weigelt 1977; Weigelt \& Wirnitzer 1983; Lohmann et al. 1983; Hofmann \& Weigelt 1986).

For HK Ori, photometric calibration in the $K^{\prime}$-band was carried out by observing the photometric standard stars HD 18881 and Gl 105.5, chosen from Elias et al. (1982), on October 19, 1997. For the photometric calibration in the H-band the photometric standard star HD 40335 and the star HD 31648 were observed on October 22 and 19, 1997. The photometric calibration in the visible is based on our speckle observations of NX Pup from March 10, 1995 (Schöller et al. 1996). Simultaneously to our observations of NX Pup, CCD photometry of the unresolved pair NX Pup A/B was carried out at the Danish $1.5 \mathrm{~m}$ telescope at La Silla. Observations of standard stars taken from the list by Landolt (1992) allowed for the absolute photometric calibration.

\section{Results}

Each of the observed visibility functions clearly showed a pattern suggestive of a binary system. The separation and position angle of the system were measured directly from the two dimensional visibility function. Maps of the objects were made and the flux ratios were also measured. The recovered parameters for the various systems are listed in Table 1. Below, we discuss each object individually.

\section{1. $L k H \alpha 198$}

LkH $\alpha 198$ (SIMBAD coordinates: $\alpha=00^{\mathrm{h}} 11^{\mathrm{m}} 25.97^{\mathrm{s}}, \delta=$ $+58^{\circ} 49^{\prime} 29.1^{\prime \prime}, \mathrm{J} 2000$ ) is a well-studied HAeBe located in the dark cloud Lynds 1265 at a distance of about 600 pc. It is associated with another well-known HAeBe star, V376 Cas, which lies approximately $35^{\prime \prime}$ to the north. A schematic picture of the complex environment around these two sources is given in the paper by Koresko et al. (1997). We reproduce a previously unpublished HST image from the archives in Fig. 1, which shows the inner $15^{\prime \prime} \times 15^{\prime \prime}$ of the system. Other images of the inner region of the system are shown in Koresko et al. (1997), Fukagawa et al. (2002) and Perrin et al. (2004).

Lagage et al. (1993) detected an embedded source ( $\mathrm{LkH} \alpha 198$ B) 6" north of $\mathrm{LkH} \alpha 198$ in $10 \mu \mathrm{m}$ images which is also visible at $2.2 \mu \mathrm{m}$ ( $\mathrm{Li}$ et al. 1994) and in the $1.1 \mu \mathrm{m}$ HST image shown here. A deeply embedded protostar (LkH $\alpha 198 \mathrm{MM}$ ) was found about 19" to the northwest by Sandell \& Weintraub (1994) using the JCMT at $800 \mu \mathrm{m}$. This object dominates in the millimetre and submillimetre but is not visible at all at shorter wavelengths. The most striking extended feature in the optical is a large bubble-like elliptical loop extending some $40^{\prime \prime}$ to the southeast at a position angle of approximately $135^{\circ}$ (Corcoran et al. 1995). Coronagraphic imaging in the near-infrared by Fukagawa et al. (2002) showed in more detail the close environment of $\mathrm{LkH} \alpha$ 198. Their image showed the primary extended along a roughly north-south axis $\left(\mathrm{PA}=15^{\circ}\right)$. This elongation was also reported by Koresko et al. (1997), and is visible in the HST image in Fig. 1, but here the issue is complicated by the presence of a diffraction spike along the same axis. 
Table 1. Summary of the observations. For each observation, the epoch and observing wavelength is shown, together with the recovered binary separation (in both mas and AU for the assumed distance), position angle (measured anticlockwise from north), and flux ratio (typical uncertainty 10-20\%). Where photometric calibration was performed, the individual magnitudes of the components are also given.

\begin{tabular}{|c|c|c|c|c|c|c|c|c|}
\hline \multicolumn{9}{|c|}{ 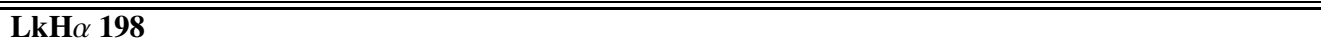 } \\
\hline Epoch & $\begin{array}{c}\lambda_{c} \\
{[\mathrm{~nm}]}\end{array}$ & $\begin{array}{c}\Delta \lambda \\
{[\mathrm{nm}]}\end{array}$ & $\begin{array}{c}\rho \\
\text { mas }\end{array}$ & $\begin{array}{c}\rho \\
\mathrm{AU}\end{array}$ & PA & $m_{A}$ & $m_{B}$ & $\overline{F_{s} / F_{p}}$ \\
\hline 1996.75 & 2191 & 411 & $66.3 \pm 4$ & 39.8 & $242 \pm 2$ & & & 0.25 \\
\hline 1997.79 & 2165 & 328 & $66.6 \pm 3$ & 40.0 & $242 \pm 2$ & & & 0.20 \\
\hline 1998.45 & 2110 & 192 & $65.8 \pm 3$ & 39.5 & $245 \pm 2$ & & & 0.20 \\
\hline 1999.74 & 1648 & 317 & $63.9 \pm 2$ & 38.3 & $246 \pm 1$ & & & 0.17 \\
\hline 2001.83 & 2115 & 214 & $58.4 \pm 3$ & 35.0 & $250 \pm 1$ & & & 0.16 \\
\hline 2002.73 & 1648 & 317 & $50.4 \pm 2$ & 30.2 & $255 \pm 2$ & & & 0.16 \\
\hline 2003.78 & 2115 & 214 & $48.6 \pm 3$ & 29.2 & $253 \pm 2$ & & & 0.27 \\
\hline \multicolumn{9}{|l|}{ Elias 1 } \\
\hline 1996.75 & 2191 & 411 & $50.5 \pm 4$ & 7.07 & $54 \pm 3$ & & & 0.69 \\
\hline 2003.76 & 2115 & 214 & $60.4 \pm 1$ & 8.46 & $59 \pm 1$ & & & 1.00 \\
\hline \multicolumn{9}{|l|}{ HK Ori } \\
\hline 1995.18 & 550 & 60 & $334.2 \pm 11.5$ & 153.72 & $44.1 \pm 1.5$ & $12.13 \pm 0.2$ & $13.01 \pm 0.2$ & 0.45 \\
\hline 1995.18 & 656 & 60 & $333.4 \pm 11.5$ & 153.35 & $44.6 \pm 1.5$ & $11.62 \pm 0.2$ & $12.53 \pm 0.2$ & 0.43 \\
\hline 1997.80 & 1613 & 304 & $339.2 \pm 6.2$ & 156.03 & $43.8 \pm 0.5$ & $8.55 \pm 0.14$ & $9.76 \pm 0.16$ & 0.33 \\
\hline 1997.80 & 2165 & 328 & $343.1 \pm 6.8$ & 157.83 & $44.2 \pm 0.5$ & $7.42 \pm 0.16$ & $9.65 \pm 0.17$ & 0.13 \\
\hline 2003.78 & 2115 & 214 & $347.7 \pm 2.5$ & 159.95 & $41.8 \pm 0.7$ & & & 0.17 \\
\hline \multicolumn{9}{|c|}{ V380 Ori } \\
\hline 2003.78 & 2115 & 214 & $122.7 \pm 2.5$ & 56.44 & $224.0 \pm 2.0$ & & & 0.27 \\
\hline
\end{tabular}

Numerous $\mathrm{HH}$ objects are found around $\mathrm{LkH} \alpha 198$ (see e.g. Corcoran et al. 1995; Molinari et al. 1993; Goodrich 1993). Corcoran et al. (1995) found knots of SII emission tracing both sides of a jet from $\mathrm{LkH} \alpha 198$ at a position angle of around $160^{\circ}$. a further knot of emission was seen along the same axis on the opposite side of $\mathrm{LkH} \alpha 198$ at position angle $340^{\circ}$. These objects have been designated HH 164. Spectra of these emission knots revealed that they have a low radial velocity, and therefore suggest that the jet lies close to the plane of the sky. Another knot seems to trace the southeast part of a jet apparently originating from the companion $\mathrm{LkH} \alpha 198 \mathrm{~B}$ along the axis of the loop. This knot, together with [SII] emission at the loop apex, has been designated HH 161. The trail of nebulosity that can be seen extending eastwards from the previouslyknown companion $\mathrm{LkH} \alpha 198 \mathrm{~B}$ may be the northern edge of an outflow cavity caused by the flow from this source driving HH 161. Nebulosity is also seen extending east from the main object. This region contains "streamer"-like structure with the same position angle as HH 161 and this might indicate interaction of the outflow from $\mathrm{LkH} \alpha 198 \mathrm{~B}$ with material surrounding the primary. Aspin \& Reipurth (2000) discovered a further $\mathrm{HH}$ object, $\mathrm{HH} 461$, with a faint bowshock morphology, along the axis of the $\mathrm{LkH} \alpha 198$ jet some $80^{\prime \prime}$ to the southeast. McGroarty $\&$ Ray (2003) identified two further bowshock-shaped structures, designated HH 801 and HH 802, which lie along the line of HH 164 with a total separation of $2.3 \mathrm{pc}$.

Polarization maps in the $I$ band by Leinert et al. (1991) and Piirola et al. (1992), and at $V$ and $I$ by Asselin et al. (1996) all show a centrosymmetric polarization pattern extending in an arc to the southeast. The map of Piirola et al. (1992), which was taken under conditions of extremely good seeing, also shows a similar centrosymmetric pattern extending to the northwest.
A region of polarization perpendicular to the centrosymmetric pattern was seen within the central 0.'5. This was taken to be the signature of a central, unresolved disk. Perrin et al (2004) used laser guide star adaptive optics to obtain very high quality near-infrared $J, H$ and $K_{\mathrm{s}}$ band polarimetry of the central part of the $\mathrm{LkH} \alpha 198$ system. They saw a polarized biconical nebula aligned almost exactly north-south, with a dark unpolarized central region. This biconical nebula, which can be interpreted as scattering from an evacuated bipolar cavity caused by an outflow, is consistent with $\mathrm{LkH} \alpha 198$ being the source of the outflow HH164, but the axis is apparently misaligned by approximately $20^{\circ}$.

Our speckle observations of $\mathrm{LkH} \alpha 198$ span seven years, from 1996.75 to 2003.78 . The two dimensional visibilities show clear signatures of binarity, and the position of the companion, which we designate $\mathrm{LkH} \alpha 198 \mathrm{~A} 2$, relative to the primary ( $\mathrm{LkH} \alpha 198 \mathrm{~A} 1)$ changes significantly over the period of the observations. The track of the secondary relative to the primary is shown in Fig. 2. The trajectory shows signs of curvature to the east, indicating that we are seeing a segment of an orbit, although it appears a straight line trajectory cannot be excluded. A map of the system reconstructed from the 1999 observations, is also shown in Fig. 2.

\subsubsection{Orbital motion}

With only seven points, spanning a short segment of the apparent orbit, it is not possible to determine the true orbit with any certainty. Nevertheless, we make some preliminary orbital fits and explore the constraints which can be imposed on the motion. 


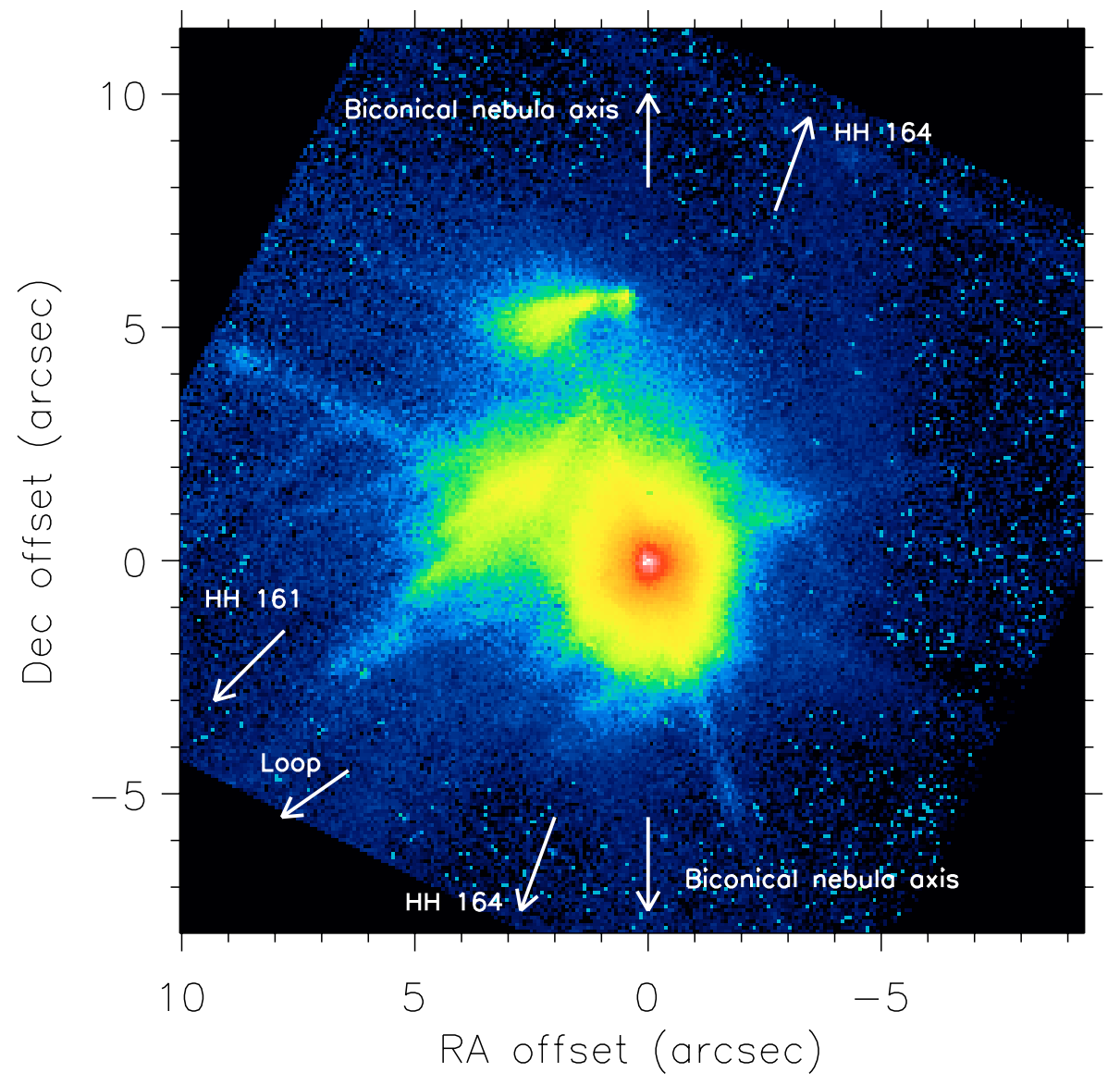

Fig. 1. HST image of $\operatorname{LkH} \alpha 198$, taken with the NICMOS camera at $1.1 \mu \mathrm{m}$. North is up and East to the left. The source approximately $6^{\prime \prime}$ due north of $\mathrm{LkH} \alpha 198$ is the already known companion $\mathrm{LkH} \alpha 198 \mathrm{~B}$. A fan-shaped trail of emission is visible to the east of this companion. The new speckle companion, $\mathrm{LkH} \alpha 198 \mathrm{~A} 2$, lies within 60 mas of the primary and is not discernable in this image (see Fig. 2). The directions to various associated $\mathrm{HH}$ objects and other structures are indicated. The various structures comprising HH 164 were identified by Corcoran et al. (1995) as the jet of $\mathrm{LkH} \alpha 198$. The HH object $\mathrm{HH} 161$ is believed to be powered by the companion $\mathrm{LkH} \alpha 198 \mathrm{~B}$, and the arrow pointing to this object is shown originating from the position of this object. Interesting streamer-like structure can be seen to the east of LkH $\alpha 198$ with the same position angle as HH 161, perhaps indicating that the HH 161 flow interacts with the material around LkH $\alpha$ 198. The axis of the biconical nebula mapped by Perrin et al. (2004) is indicated, running nearly exactly North-South. The opening angle of this structure is approximately $30^{\circ}$, and so the outflow HH 164 emerges from within the supposed bipolar cavity, even though its direction is not exactly aligned with the axis.

The exact configuration of the orbit is particularly interesting in the case of $\mathrm{LkH} \alpha 198$ because of the complex environment of the system. The outflow has a low radial velocity, suggesting that it lies in the plane of the sky and that the inner disk should be seen nearly edge-on. This is also the impression given by the polarization maps of Perrin et al. (2004). Longterm photometric monitoring by Mel'nikov (1997) shows that $\mathrm{LkH} \alpha 198$ lacks the strong photometric variations which define UXOR sources, despite the supposed edge-on viewing angle of any disk. According to the prevailing theories of UXOR variability, in which the deep photometric minima are caused by obscuration by clumps in a disk, this makes the object something of an anomaly. The presence of the companion and the details of the orbit are interesting in the context of the complex environment, and we therefore make a more detailed discussion of the orbit than would be justified for an isolated system with such a short known orbital segment.

We made a number of fits to the observed relative motion of the $\mathrm{LkH} \alpha 198$ companion. The fits were made numerically by running a 2-body code multiple times and minimizing the resulting deviations from the points. Conventional orbital parameters for the best fit, such as the major axis, eccentricity and so on, can be recovered from a full simulation of the bestfit orbit.

The mass of the primary was estimated by Hillenbrand et al. (1992) to be $1.6 M_{\odot}$ based on the luminosity estimated from the dereddened $V$ magnitude and a spectral type of A5. However, Natta et al. (1992) estimated the luminosity to be $250 L_{\odot}$ from far infrared measurements, which by comparison with pre-main-sequence tracks from Palla \& Stahler (1993), suggests a mass of 3.5-4 $M_{\odot}$, if the bulk of this luminosity can be assumed to originate from the primary. If half this luminosity arises from accretion power, the estimated primary mass would fall to 3.0-3.5 $M_{\odot}$. For an orbit calculation, to the mass of the primary must be added the mass of any compact circumstellar structure such as a disk. Leinert et al.'s (1991) estimate of the mass of the immediate circumstellar material was only $0.01 M_{\odot}$, and so we neglect the possible mass of circumprimary 

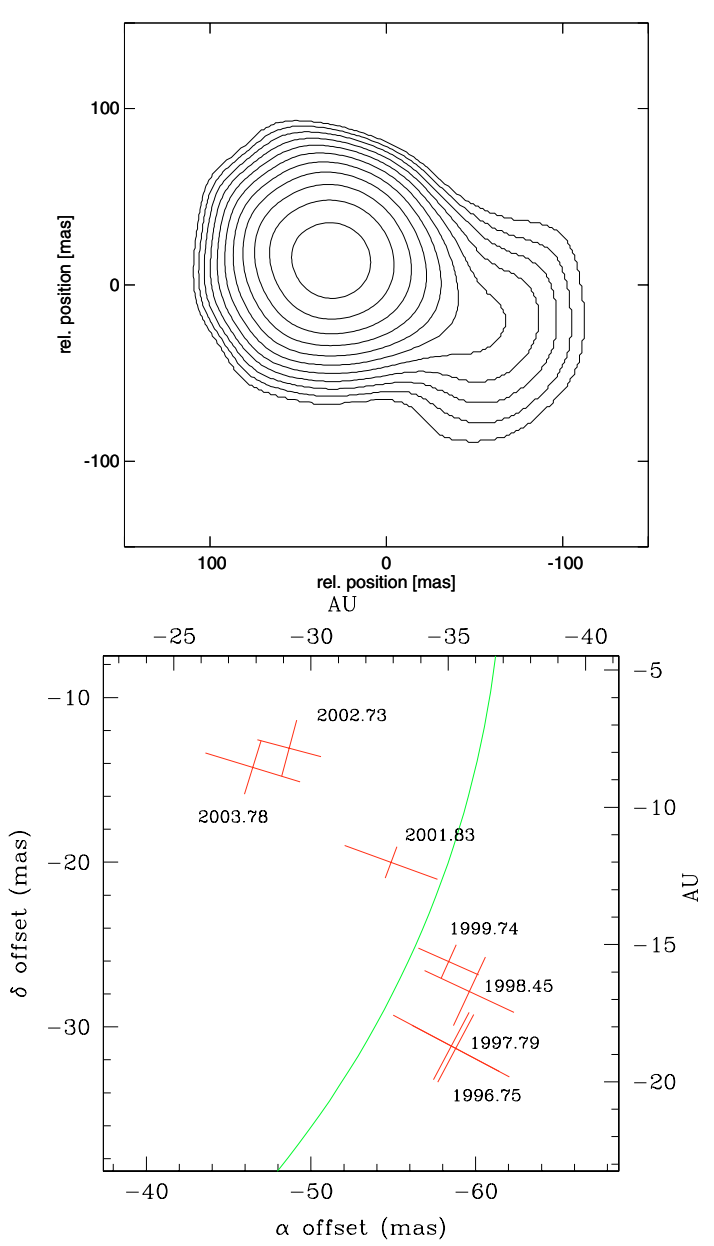

Fig. 2. Top: contour map of the $\mathrm{LkH} \alpha 198$ system constructed from speckle observations on the 26th October 1999. Bottom: the position of the $\mathrm{LkH} \alpha 198$ companion relative to the primary. The scale is given in mas and $\mathrm{AU}$ for an assumed distance of $600 \mathrm{pc}$. The solid line is a circle of radius $37 \mathrm{AU}$, for an assumed distance of $600 \mathrm{pc}$, centred on the primary

and circumsecondary material. The flux ratio of the secondary to the primary is measured to be 0.20 at $K^{\prime}$ and 0.16 at $H$ (see Table 1). If this is taken as a rough estimate of the luminosity ratio, the mass of the secondary is estimated from evolutionary tracks to be either approximately $1.0 M_{\odot}$ if the primary is $1.6 M_{\odot}$ or $2-2.5 M_{\odot}$ if the primary is around $3.5-4 M_{\odot}$. For the sake of fitting the orbit, we have adopted two different masses for the system, one of 1.6 and $1.0 M_{\odot}$, following Hillenbrand et al. (1992), and one of 4.0 and $2.5 M_{\odot}$, following Natta et al. (1992).

The motion on the sky leads to tight constraints on the motion in the plane of the sky. Because such a short segment of the orbit is observed, however, there is little constraint on the possible initial position and velocity in the radial direction. On the sky, the secondary covers an apparent distance of almost $12 \mathrm{AU}$ in a time of six years. This implies a velocity in the plane of the sky of $9.5 \mathrm{~km} \mathrm{~s}^{-1}$. For a system comprising a $1.6 M_{\odot}$ and $1.0 M_{\odot}$ components, the escape velocity would be approximately $11 \mathrm{~km} \mathrm{~s}^{-1}$. This implies that, if the system has such a low mass, the orbit must lie close to the plane of the sky (assuming it is in fact bound). On the other hand, for a system with a total mass of $6.5 M_{\odot}$, the escape velocity is approximately $17 \mathrm{~km} \mathrm{~s}^{-1}$, so that the true velocity can be substantially larger than the apparent velocity on the plane of the sky, and the orbit can be significantly inclined to the line of sight.

In Fig. 3, we show four fitted orbits for various assumed system parameters. The orbital parameters and fit $\chi^{2}$ values for these are listed in Table 2. The top left panel shows the fit for a system with masses 1.6/1.0 $M_{\odot}$, and low values for the initial value (i.e. before the fitting procedure) of the initial radial position and velocity. This leads to a quite eccentric orbit almost in the plane of the sky. In the top right panel, we show a fitted orbit with the same low values for the initial radial position and velocity, for a system with masses $4 / 2.5 M_{\odot}$. The lower left panel shows a fit for system with $4 / 2.5 M_{\odot}$, for which the initial radial offset and velocity were chosen to attempt to produce a near-circular orbit. In fact, the eventual fit has an eccentricity of about 0.3 . Finally, a fit was made with a very large initial radial velocity. The intention of this was to incline the fit as highly as possible to the plane of the sky, so that it is as close as possible to coplanar with the supposed edge-on disk at the heart of the system. The inclination of this fit to the plane of the sky was approximately $70^{\circ}$. This fit is shown in the lower right panel of Fig. 3. Finally, a fit was made with the secondary set a long way back from the plane of the sky, and only the north-south and east-west motion varied. This produces the best-fit straightline motion, the $\chi^{2}$ of which is listed in Table 2 for comparison with the bound fits.

\subsubsection{Results of orbit fitting}

Although the straight line has the highest $\chi^{2}$ (because it can't account for the apparent curvature), there is no significant difference between the quality of the various fits. The most edge-on orbit fits which could be made had an inclination of approximately $20^{\circ}$ to the plane of the sky, and the long axis of the projected orbit was tilted by at least $20^{\circ}$ to the east-west direction. This contrasts with the Perrin et al. (2004) image which shows a disk-like structure aligned almost exactly northsouth, and the jet velocity measurements of Corcoran et al. (1995) which show low velocities for the jet material and therefore suggest that the jet lies very close to the plane of the sky. Unfortunately, without tangential velocity measurements, it is not possible to put firm limits on the inclination of the jet for comparison with the model orbits. Interestingly, the orientation of the axis of the projected orbit $(d)$ is approximately aligned with the direction of the $\mathrm{HH} 164$ jet.

\subsection{Elias 1}

Elias 1, also known as V892 Tau $\left(\alpha=04^{\mathrm{h}} 18^{\mathrm{m}} 40.60^{\mathrm{s}}, \delta=\right.$ $+28^{\circ} 19^{\prime} 16.7^{\prime \prime}, \mathrm{J} 2000$ ) is located in the Taurus-Auriga complex at a distance of approximately $140 \mathrm{pc}$ (Elias 1978). Its spectral type was estimated as A6 with $A_{\mathrm{V}}=3.9 \mathrm{mag}$ and luminosity of around $38 L_{\odot}$ (Berrilli et al. 1992), but other authors have assigned earlier spectral types of A0 (Elias 1978) or B9 (Strom \& Strom 1994). Elias 1 was classed as a type II HAeBe star by Hillenbrand et al. (1992), having a very flat or even rising SED 

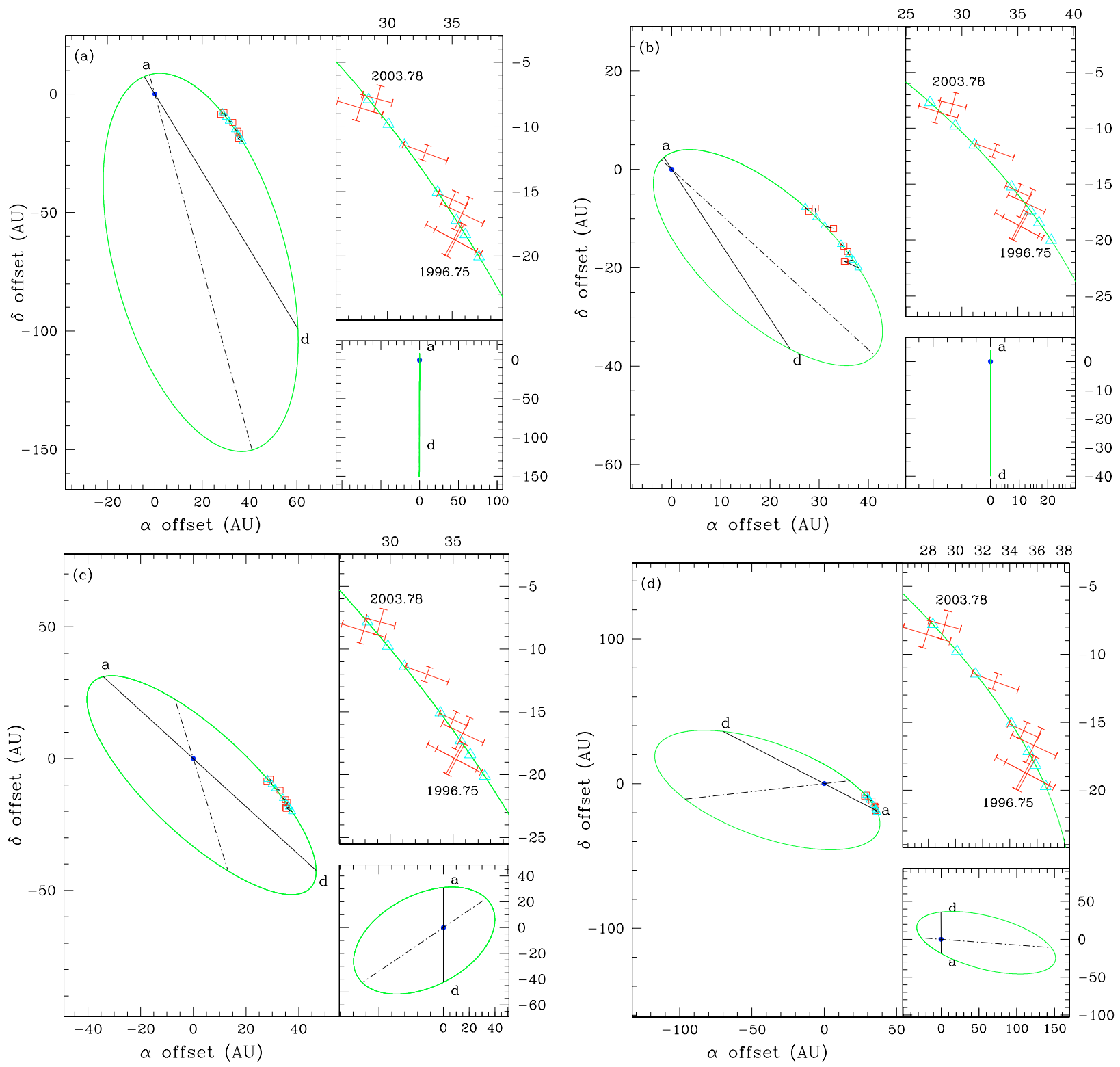

Fig. 3. Orbit fits to the motion of the LkH $\alpha 198$ companion for various choices of initial parameters. The main panel of each plot shows the fitted orbit projected on the plane of the sky. North is up and east to the left. The measured data points are plotted as open squares and the corresponding points in the fit are plotted with open triangles. A dash-dotted line marks the major axis, and a solid line marks the line of nodes (i.e. the line of intersection between the orbital plane and the plane of the sky). The ascending and descending nodes are labelled "a" and " $\mathrm{d}$ ". In the inset at upper right in each figure, we show the segment of the orbit with the data points on a larger scale. The data points are shown with errorbars, and the fitted points are again plotted as open triangles. The first and last points are labelled with the observing epoch. In the inset at lower right, we show the projection in a plane perpendicular to the plane of the sky. North is up and the radial direction (pointing away from the observer) is to the left. The coordinate systems are indicated in each panel of the top left-hand plot. a) Fit for an assumed primary mass of $1.6 M_{\odot}$ and secondary mass $1.0 M_{\odot}$. The initial guess for the fit involves a low velocity in the $R$ direction and a small displacement from the plane of the sky. As a result, the fitted orbit lies close to the plane of the sky. b) The same low starting values for $R$ position and velocity, but for an assumed primary mass of $4.0 M_{\odot}$ and secondary mass $2.5 M_{\odot}$. c) A fit with an assumed primary mass of $4.0 M_{\odot}$ and secondary mass $2.5 M_{\odot}$, with starting conditions chosen to produce a near-circular orbit. d) This fit has masses of 4 and $2.5 M_{\odot}$ and a high initial radial velocity, which is intended to produce an orbital plane as highly inclined as possible to the plane of the sky.

in the NIR to FIR. This suggests the presence of a possible envelope as well as a circumstellar disk, or possibly a flared disk. The polarization is $4.7 \%$ (Yudin 2000).

A T Tauri type companion lies approximately $4^{\prime \prime}$ to the northeast (LRH97). Kataza \& Maihara (1991) obtained one dimensional speckle interferograms at $K$ and $L$, with position angles of zero and ninety degrees. They also obtained onedimensional speckle interferograms at $L^{\prime}$ with PA $=45^{\circ}$, showing the object was resolved in this direction. The object was found to be resolved in the east-west direction, but unresolved in the north-south direction. The preferred interpretation was a flattened halo associated with a circumstellar accretion disk 
Table 2. Parameters of the various fitted orbits shown in Fig. 3. The parameters shown are the mass of the primary, mass of the secondary, major axis length, eccentricity, period, position angle of the ascending node (measured anticlockwise from north in the plane of the sky), inclination between the orbital plane and the plane of the sky, and the longitude of periastron, defined as the angle between the ascending node and the periastron measured in the plane of the true orbit and taken in the direction of the secondary's motion. All the orbits appear prograde on the sky. The last line, labelled "Straight Line", shows the $\chi^{2}$ for the best straight-line fit.

\begin{tabular}{rrrrrrrrrr}
\hline \hline Fit & $M_{1}\left(M_{\odot}\right)$ & $M_{2}\left(M_{\odot}\right)$ & Major axis (AU) & $e$ & $P(\mathrm{yr})$ & $\Omega$ & $i$ & $\omega$ & $\chi^{2}$ \\
\hline (a) & 1.6 & 1.0 & 164.2 & 0.90 & 462.7 & 31 & 0.3 & 344 & 1.32 \\
(b) & 4.0 & 2.5 & 58.8 & 0.90 & 62.7 & 33 & 0.4 & 14 & 1.37 \\
(c) & 4.0 & 2.5 & 117.9 & 0.31 & 178.0 & 48 & 70 & 300 & 1.34 \\
(d) & 4.0 & 2.5 & 206.3 & 0.69 & 412.1 & 243 & 70 & 63 & 1.15 \\
Straight Line & & & & & & & & & 1.56 \\
\hline
\end{tabular}

and extending some $40 \mathrm{AU}$ in $K$ or $100 \mathrm{AU}$ in $L$. Haas et al. (1997) later obtained 1-D specklegrams at $J, H, K$ and $L$ with a larger $3.5 \mathrm{~m}$ telescope. Their specklegrams were also oriented north-south and east-west. Mostly on the basis of the relative halo brightness at $J$ and $H$, they rejected the disk hypothesis of Kataza \& Maihara (1991) and argued instead for a bipolar nebula with east-west orientation. They also considered a possible binary interpretation, noting that a binary with position angle $45^{\circ}$ or $135^{\circ}$, separation $<0$ '. 1 and brightness ratio 0.08 at $K$ or 0.2 at $L$ was not ruled out by their data.

Elias 1 is unusual for HAeBe stars in being an X-ray source (Zinnecker \& Preibisch 1994). Giardino et al. (2004) observed a strong X-ray flare from the system, and also smaller X-ray variations which could be identified as originating on Elias 1 and not the companion Elias 1 NE. Spectral fits with a two temperature model suggested a hot component of around $k T \approx$ $2-3 \mathrm{keV}$. The time variability, and the hot plasma temperature, point to magnetically confined plasma in the neighbourhood of the star. Elias 1 is also a radio source, although its rising spectrum, though steep, is consistent with thermal emission from a wind (Skinner et al. 1993).

Our data show that Elias 1 is a close binary with a separation of approximately 50 mas and position angle approximately $50^{\circ}$ (Fig. 4). Furthermore, the relative position of the secondary with respect to the primary has changed in the seven years between the two observation epochs. The positions and flux ratios are given in Table 1 and are shown in Fig. 5.

The binarity of Elias 1 is important for the interpretation of the X-ray flux observed from this object. HAeBe stars should lack the convective zone necessary to drive a conventional dynamo. Novel dynamo mechanisms have been developed to explain HAeBe activity, such as the differential rotation dynamo model of Tout \& Pringle (1995). Also, it has been suggested that deuterium burning in a shell outside a radiative core powers a surface convective layer (Palla \& Stahler 1990). Such models are of course not necessary if a low-mass companion is in fact the source of the X-ray emission. The spectral type of A6 and luminosity of Elias 1 suggest, from comparison with tracks (Palla \& Stahler 1993), that its mass is of order 2-2.5 $M_{\odot}$. The flux ratio of the system suggests a companion mass of 1.5-2 $M_{\odot}$, which lies close to the boundary between fully radiative and partially convective stars. Therefore, it is possible that the companion has a normal convection-generated magnetic field and corona and is the source of the X-ray emission.
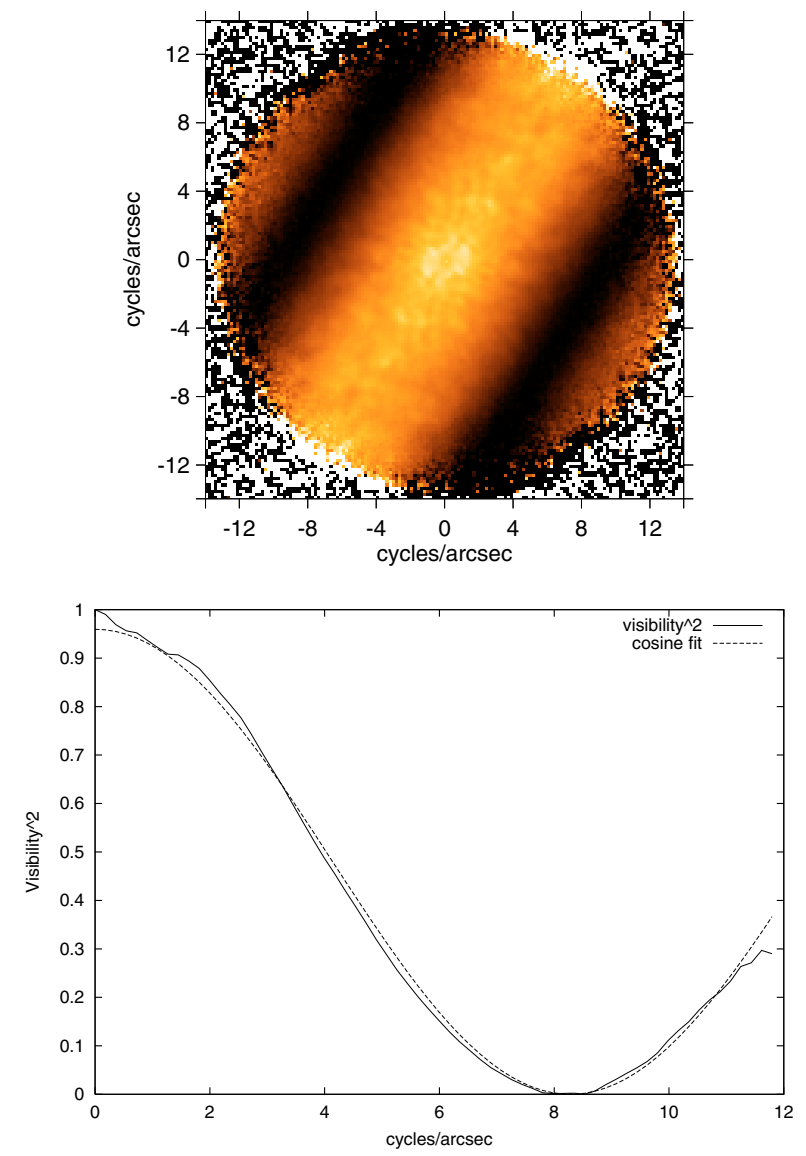

Fig. 4. Top: the $2 \mathrm{~d}$ power spectrum of Elias 1 from 2003.76. Bottom: a cut across the fringes, with a cosine fit.

\subsection{HK Ori}

\subsubsection{The data}

HK Ori ( $\alpha=05^{\mathrm{h}} 31^{\mathrm{m}} 28^{\mathrm{s}} .04, \delta=+12^{\circ} 09^{\prime} 10{ }^{\prime} 3$, J2000) is an A5 type star with $A_{\mathrm{V}}=1.2$. It was found to be a binary by LRH97, who designated the brighter NIR component to the southwest A and the fainter northeastern component B. LRH97 found a binary separation of $(0.34 \pm 0.02)^{\prime \prime}$ and position angle $(41.7 \pm 0.5)^{\circ}$. Contour maps of the system in bands $V, H$ and $K$ are shown in Fig. 6, together with 3-d plots illustrating the brightness ratio. The track of component $\mathrm{B}$ with respect to component $\mathrm{A}$ is shown in Fig. 7. Our data points trace a path from southeast to northwest. The radial position of LRH97 is broadly 

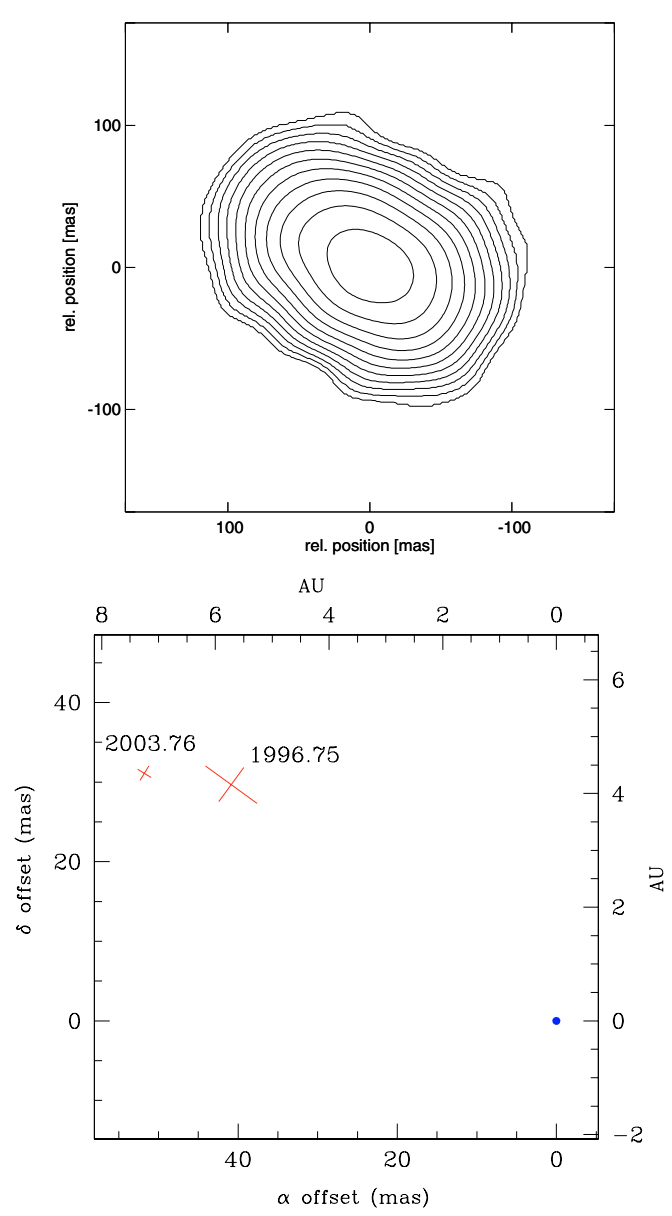

Fig. 5. Top: contour map of Elias 1 made from the 2003 observations. Bottom: the position of the Elias 1 companion relative to the primary. The primary position is marked with a dot.

consistent with our measured positions, but the azimuthal position is not in agreement with the position suggested for epoch 1992.12 from our data.

Following LRH97, we can use our flux measurements to disentagle the SEDs of the individual components of HK Ori. LRH97 used observations at wavelengths of $I^{\prime}(0.917 \mu \mathrm{m}), J$, $H$, and $K$, and had to rely on flux ratios alone. We have data at four different wavelengths, $550 \mathrm{~nm}(V), 656 \mathrm{~nm}(R)$, as well as $H$ and $K$. The optical points were not available to LRH97, and we are therefore able to separate the SEDs of the components over a significantly wider wavelength range. Furthermore, our data is photometrically calibrated (except for the 2003 point which we do not use in this analysis). There remains the problem that our measurements are not simultaneous. However, independent measurements in October, 1997 show that the $V$-magnitude was the same as during our earlier observations in $1995(V=11.7 \pm 0.1$ derived from speckle observations in 1995, $V=11.6 \pm 0.1$ in 1997, Mel'nikov private communication). This indicates that the system was in a comparable state at both epochs and that it is therefore valid to combine our data into one SED.

In Fig. 8 we present the separated SED (along with various models described in Sect. 3.3.2). SEDs for the whole system were taken from Hillenbrand et al. (1992) and Berrilli et al. (1992). An upper limit at $1.3 \mathrm{~mm}$ was taken from
Henning et al. (1997). The error bars for $U, B$ and $V$ reflect the measured variability as reported by Eiroa et al. (2002) in the $V$-band. For $R$ through $K$ the measurement uncertainty was used (typically of order 10\%), and for wavelengths longer than $2.2 \mu \mathrm{m}$, an assumed $10 \%$ uncertainty was adopted.

The SEDs of the individual components A and B are constructed from points measured by us at $V, R, H$, and $K$, and points measured by LRH97 at $I_{J}, J, H$ and $K$. Our points are calibrated as described in Sect. 2. LRH97 obtained flux ratios but not absolute fluxes. We therefore normalised their values against the total flux given by Hillenbrand et al. (1992). In the case of LRH97's $I_{J}$ point, the Cousins system flux of the composite SED was converted to a supposed Johnson system $I$ flux using the information given in Bessell (1983).

All fluxes were corrected for the effect of interstellar reddening. The visual extinction, $A_{\mathrm{V}}=1.2$ was determined by Hillenbrand et al. (1992) from the $(B-V)$ excess. The extinction law given by Cardelli et al (1989) was used to estimate the wavelength dependency of the extinction, with the assumption that $R_{V}=3.1$.

The SED is clearly dominated by component A (which we designate the primary) at longer wavelengths. The brightness ratio moves towards unity in the very near-infrared ( $I$ and $J$ bands). LRH97 considered one possible scenario in which the brightest infrared component, A, might in fact be a low-mass IR companion, and the fainter component the primary. This conjecture was apparently confirmed by Baines et al. (2004) who used a spectroastrometry technique to show that the southwestern component dominates the emission at $\mathrm{H} \alpha$, Their data also suggested that the northeastern component might be the brightest optical source in the system. However, our data points at $550 \mathrm{~nm}$ and $656 \mathrm{~nm}$ now exclude this possibility, and show clearly that the brighter IR component to the southwest also dominates in the optical (see top panel of Fig. 7). Variability of the T Tauri source is a possible but not convincing explanation for this discrepency, since the photometrically calibrated flux of component A matches the composite SED published in Hillenbrand et al. (1992), whereas the flux of component B does not.

The infrared colours of the two components are very different. The brightest component has $H-K=2.12$, whereas component B has $H-K=1.10$. The implication is that component A possesses significantly more circumstellar material than component $\mathrm{B}$, which resembles a naked photosphere of around $4000 \mathrm{~K}$. The positions of the two objects are shown in a colour-colour diagram in Fig. 9. Comparing this figure to colour-colour diagrams of young stars, for example Fig. 15 of Hillenbrand et al. (1992), it is clear that the primary (component $\mathrm{A}$, to the southwest) occupies a typical position for an intermediate mass young star, whilst the companion (component $\mathrm{B}$, to the northeast) occupies a position typical for a T Tauri star.

We have fitted a $4000 \mathrm{~K}$ photosphere, corresponding to a mid-K spectral type, to the SED of component B. This fit represents the SED of component $B$ very well, with the possible exception of the $B$-band point. Where no resolved points were available, we have subtracted this fit to determine points for A. In practice, this model subtraction makes little difference to 

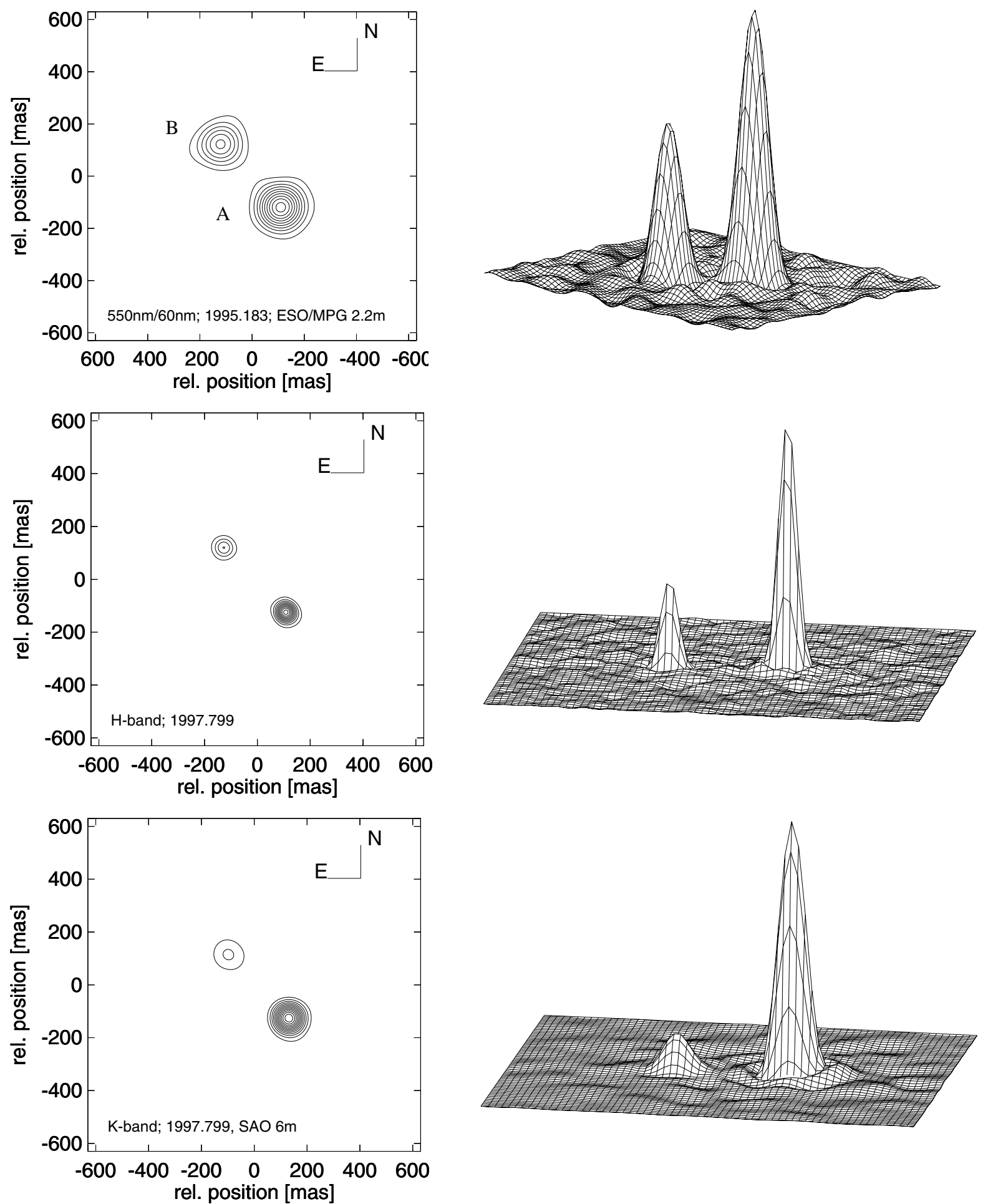

Fig. 6. Bispectrum speckle interferometry reconstructions of HK Ori. Contour plots on the left show the relative positions of the sources and wire-frame plots on the right illustrate the relative brightnesses. The components A and B are identified in the topmost contour plot.

most of the fluxes measured for the composite system, and this method is nearly equivalent to assuming that component A contributes essentially all the flux at $U, B$ and in the mid-infrared.

\subsubsection{Modelling strategy}

Component A is responsible for most of the IR-excess of the system and presents a "double-bumped" SED. The first bump peaks around $400 \mathrm{~nm}$ and is produced by the central A-type star. The second one, peaking around $3 \mu \mathrm{m}$, is a signature of the presence of circumstellar matter and is usually interpreted as emerging from an accretion disk (e.g. Hillenbrand et al. 1992).

Let us emphasise that the NIR- and MIR-excesses are unusually strong for a Herbig star, with a flux intensity $\lambda F_{\lambda}$ peaking as high as the stellar photosphere (see Fig. 8) while it is one order of magnitude smaller in most HAeBes (see Fig. 1 in 
$\mathrm{AU}$

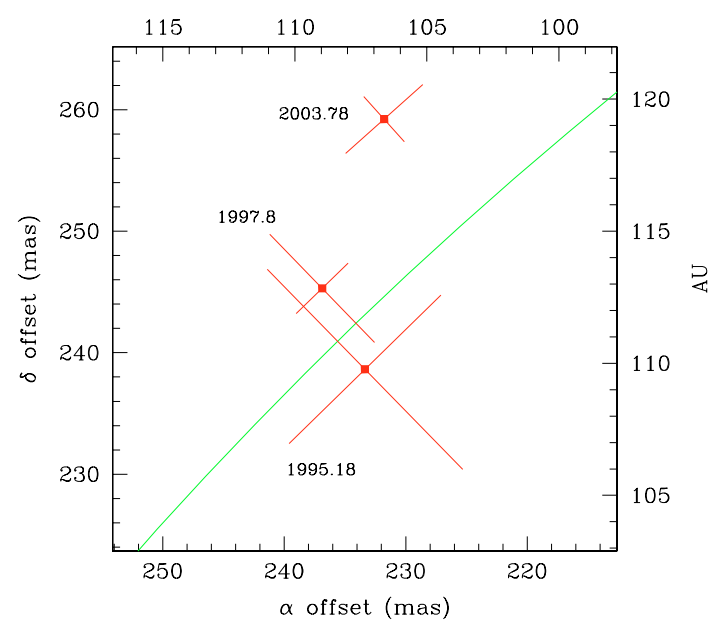

Fig. 7. The position of the HK Ori companion B relative to the primary. The separate optical positions from 1995 and separate $H$ and $K$ positions for epoch 1997.8 have each been averaged to produce one position for each date. The solid line is a circle of radius $155 \mathrm{AU}$ for an assumed distance of $460 \mathrm{pc}$ centred on the primary.

Natta et al. 2001). Making a simple three blackbody fit, we estimate that the circumstellar flux accounts for fully two thirds of the observed flux from component $\mathrm{A}$. The low visual extinction $\left(A_{\mathrm{V}}=1.2\right)$ hints that the star is not significantly shadowed by circumstellar material, so that the circumstellar matter actually emits twice as much flux as the star in the observer's direction. Higher values of $A_{\mathrm{V}}$ for the photosphere can be considered if the extinction law is taken to be non-standard $\left(R_{V} \sim 5\right.$ leads to $A_{\mathrm{V}} \sim 3$ for the measured $E(B-V)$ ), but we feel this is unlikely since the the derived extinction is in agreement with the extinction from interstellar matter measured for nearby Herbig-Haro objects (Goodrich et al. 1993). Though the reprocessing of stellar light is widely accepted to be the main source of energy in typical HAeBes (Chiang \& Goldreich 1997; Natta et al. 2001; Dullemond et al. 2001), it seems unable to account for so high a IR-excess: an optically thin envelope would reprocess only a fraction $\tau \ll 1$ of the stellar light, and an optically thick disk $\approx h / r \sim 0.05-0.5$, where $h$ is the thickness of the disk, and $r$ the distance to the star. So, we follow Hillenbrand et al. (1992) in their assumption that viscous heating (i.e. accretion luminosity) is responsible for most of the observed flux. We use a generalised version of the Chiang \& Goldreich (1997) model by Lachaume et al. (2003), that includes viscous dissipation in addition to stellar irradiation, to fit the SED. Our approach has the advantage of not excluding a priori the hypothesis of an irradiated disk.

The fitted parameters are summarised in Table 3 , and the SED data and fit are displayed in Fig. 8. The large IR excess at $\lambda \gtrsim 2 \mu \mathrm{m}$ cannot be explained with irradiation only (dash-dotted line in Fig. 8) and requires a high accretion rate $\dot{M}=2.5 \times 10^{-6} M_{\odot} \mathrm{yr}^{-1}$ (solid line). The depression in the SED around $1 \mu \mathrm{m}$ suggests that the inner, hot regions of the disk should be depleted. which our model backs: the inner rim of the disk is located at $31 R_{\odot}=20 R_{*}=0.15 \mathrm{AU}$ from the star.
On one hand, the accretion rate and outer disk truncation radius are in good agreement with those derived by Hillenbrand et al. (1992). This could be expected, since these parameters are sensitive to the mid- and far-IR SED that was not affected by our resolving the binary. On the other hand, the visible and NIR SED for component A significantly differs from that of the total system, so that our estimates for the stellar photosphere (derived from the visible photometry) and the inner truncation (sensitive to the depression at $1 \mu \mathrm{m}$ ) are quite different from their values.

\subsubsection{Disk properties}

The model was computed with the viscosity coefficient $\alpha=$ $2 \times 10^{-2}$ (see Shakura \& Sunyaev 1973), but choosing any $\alpha$ above $10^{-3}$ does not greatly affect the quality of the fit. The upper limit at $1.3 \mathrm{~mm}$ constrains the amount of material at larger radii, and the mass of the disk is limited to about $0.06 M_{\odot}$. Combined with the high accretion rate, this estimate leads to a lifetime of the disk between a few $10^{4}$ and a few $10^{5}$ years, i.e. less than the typical age of HAeBe stars. On one hand, the lifetime may be extended by replenishing of the disk with material from some wider reservoir. The scenario of a circumbinary disk is unlikely, since material with high angular momentum should accrete on to the secondary instead of the primary, at least in a coplanar system. This favors the hypothesis of mass infall from a spherically symmetric envelope. This envelope should however comprise at least a few solar masses, implying a significant reddening, which is not observed. On the other hand, HK Ori could be in a transitory high-accretion state of an otherwise "quiescent", passive accretion disk.

More concerning is the presence of a large central gap $\left(20 R_{\star}\right)$ in an intensively accreting disk. As pointed out by Hartmann et al. (1993), a strongly accreting disk should not present such a gap: the gap is either understood as a drop in optical thickness beyond the dust sublimation radius, or as a cleared region due to stellar magnetic fields which drive the matter into accretion streams or into an outflow. However, the large amount of material in this disk would ensure that the gas remains optically thick, whilst the field strength required to truncate the disk at $20 R_{*}$ would be of order tens of kilogauss. Though these arguments plead for a moderately accreting disk, we were not able to find a mechanism other than accretion that could account for the very large IR-excess. In particular, the reprocessing of the stellar light leads to IR fluxes of up to a few tens of percent of that of the star, as previous simulations by Chiang \& Goldreich (1997) and Dullemond et al. (2001) suggest.

\subsection{V380 Ori}

V380 Ori ( $\left.\alpha=05^{\mathrm{h}} 36^{\mathrm{m}} 25.43^{\mathrm{s}}, \delta=-06^{\circ} 42^{\prime} 57.7^{\prime \prime}, \mathrm{J} 2000\right)$ is a B9 star in the Orion complex at a distance of $460 \mathrm{pc}$. It was classified as a group I source by Hillenbrand et al. (1992), meaning that its SED can be fitted by a geometrically thin, optically thick accretion disk. 


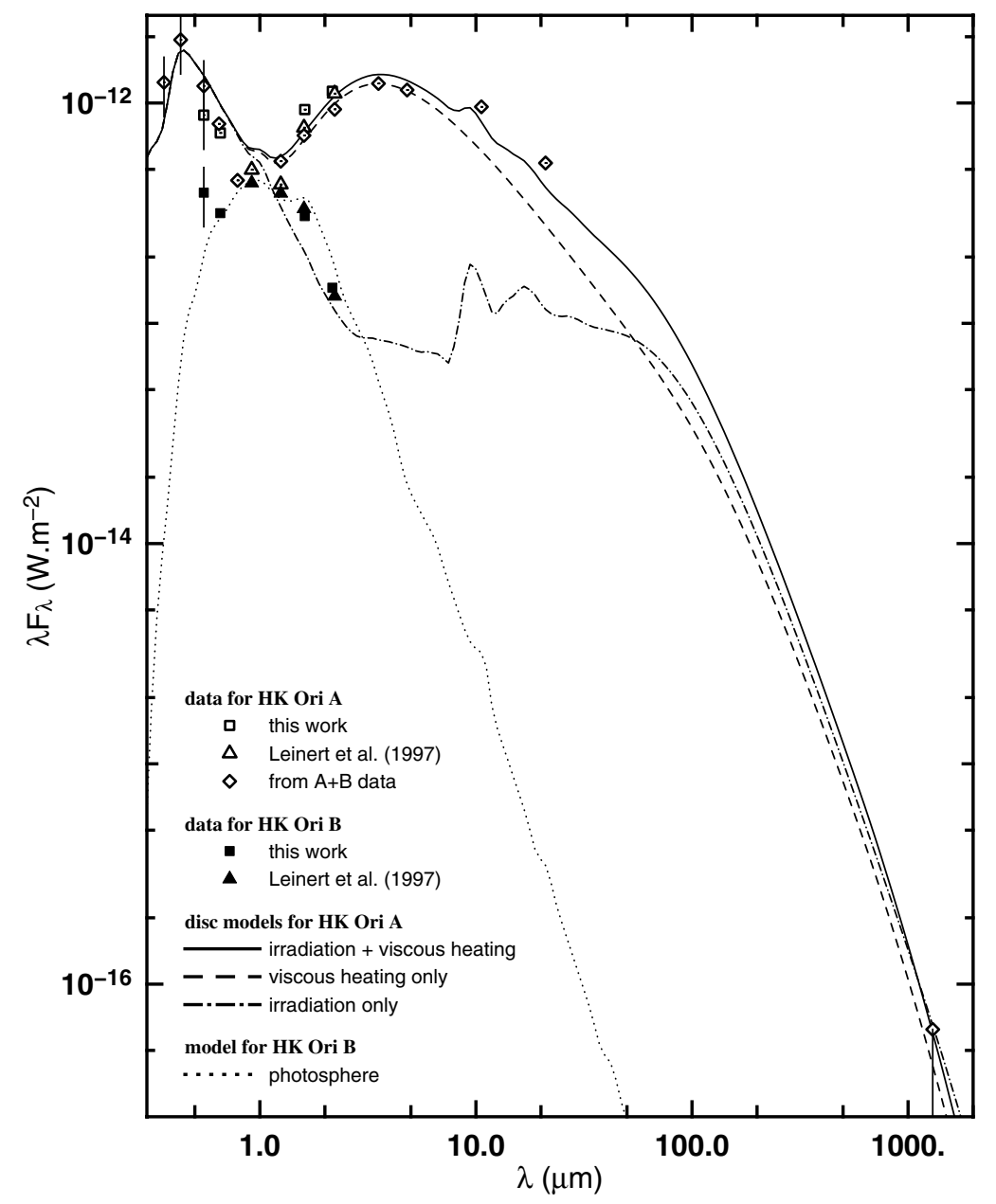

Fig. 8. The SED of HK Ori's components. Data: open symbols stand for the primary and filled ones for the secondary. Separate photometric measurements for HK Ori A and HK Ori B from this work are marked with squares. Flux ratios measured by LRH97 and normalised to separate fluxes by reference to the composite photometry of Hillenbrand et al. (1992) are marked with triangles. For other points (diamonds), we have subtracted the model fit to HK Ori B from the total system flux as measured by Hillenbrand et al. (1992) to obtain supposed values for the flux of HK Ori A. We also show the $1.3 \mathrm{~mm}$ upper limit obtained by Henning et al. (1998), also with the flux of the HK Ori B model subtracted. In $U, B$, and $V$, the uncertainties result from the reported variability. At NIR wavelengths, the measurement uncertainties are of order $10 \%$ and are not plotted for the sake of clarity. Model for HK Ori A: a two-layer disk model by Lachaume et al. (2003), including irradiation by the central star and heating by viscosity fits the data very well at all wavelengths (solid line). For comparison's sake similar models, in which irradiation or viscous heating is turned off, are displayed (dashed line is without irradiation and dash-dotted line is without viscous heating). Model parameters are shown in Table 3. Model for HK Ori B: the SED of the secondary does not hint at an infrared excess, so a photosphere model is fit to the data (dotted lines). It should be noted that excess emission from distant reprocessing material cannot be ruled out.

The system was resolved as a $\sim 150$ mas binary by LRH97. They attempted to divide the SED of the system into the contributions of the two components. As with HK Ori, they considered two models; one in which the brightest source in the IR continues to dominate in the optical, and one in which the brighter IR source is an "IR companion" which is less bright in the optical. Millan-Gabet et al. (2001) used long-baseline interferometry to resolve V380 Ori A. Their uniform ring model fit had an inner radius of 2.5 mas, corresponding to just over $1 \mathrm{AU}$.

In Fig. 10 we show a contour plot of the V380 Ori system, and a plot of the position we obtained for the secondary in 2003, together with the position obtained by LRH97.

Based on the data presented, it would appear that there has been orbital movement of the V380 Ori system. The relative position of the secondary has changed by about $26 \mathrm{AU}$. This apparent shift has been largely in a tangential sense, as can be seen by comparison with the circle segment shown in the figure.

The flux ratio at $K^{\prime}$ is measured by us to be $0.266 \pm 0.008$, whereas LRH97 obtained $0.35 \pm 0.08$. These values are almost compatible with each other to within $1 \sigma$. Unfortunately, unlike in the case of HK Ori, we have no optical observation and so cannot resolve the ambiguity considered by these authors in the SEDs.

\section{Conclusions}

We have used bispectrum speckle interferometry to resolve for the first time the binary companions to the HAeBe stars 


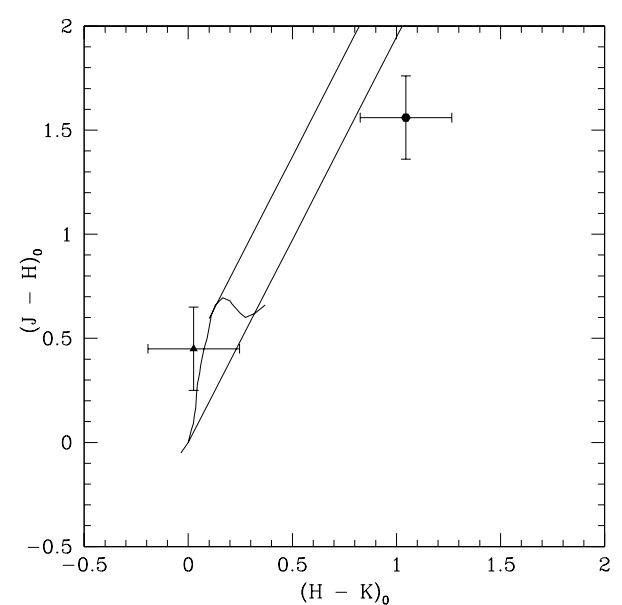

Fig. 9. $(H-K)$ vs. $(J-H)$ colour-colour diagram showing the positions of the primary and secondary in the HK Ori system. Component A is shown with a circular point and component $\mathrm{B}$ with a triangle. The colours have been corrected for the effects of interstellar reddening. The main sequence (from Bessell \& Brett 1988) and the direction of interstellar reddening are also shown.

Table 3. Fitted parameters for HK Ori. A disk mass of $0.06 M_{\odot}$ is derived from these parameters. The parameters obtained by Hillenbrand et al. (1992) are given in italic when available.

\begin{tabular}{lcl}
\hline \hline \multicolumn{3}{c}{ Component A } \\
stellar radius $\left(R_{\odot}\right)$ & 1.55 & $1.7^{d}$ \\
stellar mass $\left(M_{\odot}\right)$ & 2.0 & $2.0^{d}$ \\
stellar temperature $(\mathrm{K})$ & 8500 & $9700^{d}$ \\
\multicolumn{3}{c}{ Component B } \\
stellar radius $\left(R_{\odot}\right)$ & 4.1 \\
stellar mass ${ }^{a}\left(M_{\odot}\right)$ & 1.0 & \\
stellar temperature $(\mathrm{K})$ & 4000 \\
\multicolumn{1}{c}{$\mathrm{Disk}$ around $\mathrm{A}$} \\
disk inner radius $\left(R_{\odot}\right)$ & 31 & 17 \\
disk outer radius $(\mathrm{AU})$ & 30 & 29 \\
accretion rate $\left(M_{\odot} \mathrm{yr}^{-1}\right)$ & $2.5 \times 10^{-6}$ & $1.7 \times 10^{-6}$ \\
$\alpha$ & 0.02 & - \\
disk inclination $^{a}(\mathrm{deg})$ & 0 & 0 \\
distance $^{b}(\mathrm{pc})$ & 460 & 460 \\
\hline
\end{tabular}

\footnotetext{
${ }^{a}$ Kept constant.

${ }^{b}$ Assumed.

${ }^{c}$ Derived from simulation.

${ }^{d}$ Both components.
}

LkH $\alpha 198$ and Elias 1. We also resolved the known binaries HK Ori and V380 Ori. In the case of $\mathrm{LkH} \alpha$ 198, the new object lies at a separation of approximately 60 mas, or $36 \mathrm{AU}$ from the brighter component. Our observations comprise seven data points taken at intervals of approximately one year over a seven year time period. Motion of the secondary with respect to the primary is seen and the path is suggestive of an arc, although a straight line cannot be excluded. Because the companion may well have an important effect on the interesting and well-studied circumstellar environment of $\mathrm{LkH} \alpha$ 198, we have fitted several possible orbits to these points and shown
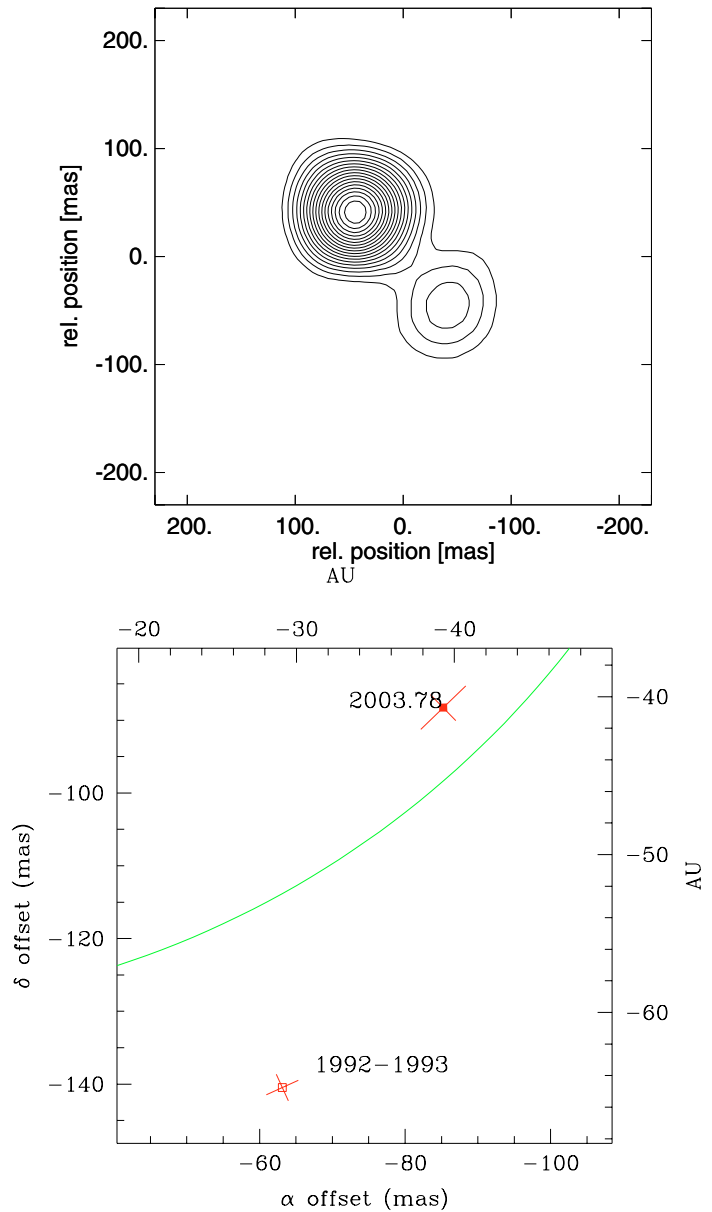

Fig. 10. Top: contour plot of the V380 Ori system. Bottom: position of the V380 Ori companion relative to the primary. The solid line is a circle of radius $60 \mathrm{AU}$ centred on the primary. The data point of LRH97 is shown with an open diamond. This position was obtained by LRH97 from data taken over a period of 3 years. Our data point is marked with a filled symbol.

that the plane of the orbit cannot be viewed exactly edge-on with an exactly east-west orientation. This may imply that the binary is not coplanar with the inferred disk powering the outflow. The flux ratio of the Elias 1 system indicates that the new companion has a mass of around $2 M_{\odot}$ and therefore may be a convective star. This opens the possibility that the X-ray emission of Elias 1 originates from the companion and is powered by conventional mechanisms. The SED of HK Ori was separated into the contributions of the primary and secondary components. The IR excess of HK Ori A was found to contribute around two thirds of the total emission from this component, suggesting that accretion power contributes significantly to the flux, in contrast to most HAeBe stars whose SEDs can often be well fitted with passive disks. We fitted an accretion disk model to the HK Ori A SED and determined the accretion rate and disk mass. From these parameters, the inferred disk lifetime is found to be quite short compared to the suspected age of HK Ori. It is possible that the disk could be replenished from a wider reservoir of material, but there are problems with such a picture; a spherical envelope should lead to extinction which is not observed, whilst in a coplanar system, accretion 
from a disk should proceed preferentially onto the secondary. Another explanation could be that the HK Ori disk is seen in an outburst phase. Such a phase need not affect the outer disk, so that the accretion rate may not be uniform throughout. Our relative positions for this system trace a path over the eight years of observations which may be the segment of an orbit. Future near-simultaneous multi-wavelength high resolution observations will allow a more detailed picture of the disk. High-resolution observations in the mid-infrared could determine whether or not component B has a passive disk. Further observations of all four stars will be necessary to build up reliable orbit determinations. $\mathrm{LkH} \alpha 198$ in particular should be a fascinating subject for future study from this point of view, since the close binary companion may be responsible for much of the complexity in the circumstellar environment of this system.

Acknowledgements. We would like to thank the anonymous referee for constructive comments which have helped us to improve the manuscript.

\section{References}

Aspin, C. \& Reipurth, B. 2000, MNRAS, 311, 522

Asselin, L., Ménard, F., Bastien, P., Monin, J.-L., \& Rouan, D. 1996, ApJ, 472, 349

Baines, D., Oudmaijer, R. D., Mora, A., et al. 2004, MNRAS, 353, 697

Berrilli, F., Corciulo, G., Ingrosso, G., et al. 1992, ApJ, 398, 254

Bessell, M. S. 1983, PASP, 95, 480

Bessell, M. S., \& Brett, J. M. 1988, PASP, 100, 1134

Cardelli, J. A., Clayton, G. C., \& Mathis, J. S. 1989, ApJ, 345, 245

Chiang, E. I., \& Goldreich, P. 1997, ApJ, 490, 368

Corcoran, D., Ray, T. P., \& Bastien, P. 1995, A\&A, 293, 550

Corporon, P., \& Lagrange, A.-M. 1999, A\&AS, 136, 429

Dullemond, C. P., Dominik, C., \& Natta, A. 2001, ApJ, 560, 957

Duquennoy, A., \& Mayor, M. 1991, A\&A, 248, 524

Elias, J. H. 1978, ApJ, 224, 857

Elias, J. H., Frogel, J. A., Matthews, K., \& Neugebauer, G. 1982, AJ, 87,1029

Eiroa, C., Oudmaijer, R. D., Davies, J. K., et al. 2002, A\&A, 384, 1038 Fukagawa, M., Tamura, M., Suto, H., et al. 2002, PASJ, 54, 969

Ghez, A. M., Neugebauer, G., \& Matthews, K. 1993, AJ, 106, 2005

Giardino, G., Favata, F., Micela, G., \& Reale, F. 2004, A\&A, 413, 669 Goodrich, R. W. 1993, ApJS, 86, 499

Haas, M., Leinert, C., \& Richichi, A. 1997, A\&A, 326, 1076

Henning, T., Burkert, A., Launhardt, R., Leinert, C., \& Stecklum, B. 1998, A\&A, 336, 565
Herbig, G. H. 1960, ApJS, 4, 337

Hillenbrand, L. A., Strom, S. E., Vrba, F. J., \& Keene, J. 1992, ApJ, 397,613

Hofmann, K.-H., \& Weigelt, G. 1986, A\&A, 167, L15

Kataza, H., \& Maihara, T. 1991, A\&A, 248, L1

Kilkenny, D., Whittet, D. C. B., Davies, J. K., et al. 1985, S. African Astron. Obs. Circ., 9, 55

Koresko, C. D., Harvey, P. M., Christou, J. C., Fugate, R. Q., \& Li W. 1997, ApJ, 485, 213

Labeyrie, A. 1970, A\&A, 6, 85

Lachaume, R., Malbet, F., \& Monin, J.-L. 2003, A\&A, 400, 185

Lagage, P. O., Olofsson, G., Cabrit, S., et al. 1993, ApJ, 417, L79

Landolt, A. O. 1992, AJ, 104, 340

Leinert, C., Haas, M., \& Lenzen, R. 1991, A\&A, 246, 180

Leinert, C., Zinnecker, H., Weitzel, N., et al. 1993, A\&A, 278, 129

Leinert, C., Richichi, A., \& Haas, M. 1997, A\&A, 318, 472 (LRH97)

Lejeune, T., Cuisinier, F., \& Buser, R. 1997, A\&AS, 125, 229

Li, W., Evans, N. J., Harvey, P. M., \& Colomé, C. 1994, ApJ, 433, 199

Lohmann, A. W., Weigelt, G., \& Wirnitzer, B. 1983, Appl. Opt. 22, 4028

Mathieu, R. D. 1992, in Complementary approaches to double and multiple star research, ed. H. A. McAlister \& W. I. Hartkopf, IAU Coll., 135, ASP Conf. Ser., 32, 30

McGroaty, F., Ray, T. P., \& Bally, J. 2004, A\&A, 415, 189

Mel'nikov, S. Y. 1997, AstLett, 23, 918

Millan-Gabet, R., Schloerb, P., \& Traub, W. A. 2001, ApJ, 546, 358

Molinari, S., Liseau, R., \& Lorezetti, D. 1993, A\&AS, 101, 59

Natta, A., Palla, F., Butner, H. M., Evans, N. J., \& Harvey, P. M. 1992, ApJ, 391, 805

Palla, F., \& Stahler, S. W. 1990, ApJ, 360, L47

Palla, F., \& Stahler, S. W. 1993, ApJ, 418, 414

Perrin, M. D., Graham, J. R., Kalas, P., et al. 2004, Science, 303, 1345

Piirola, V., Scaltriti, F., \& Coyne, G. V. 1992, Nature, 359, 399

Pirzkal, N., Spillar, E. J., \& Dyck, H. M. 1997, ApJ, 481, 392

Preibisch, T., Balega, Y., Hofmann, K.-H., Weigelt, G., \& Zinnecker, H. 1999, New Astron., 4, 531

Sandell, G., \& Weintraub, D. A. 1994, A\&A, 292, L1

Schöller, M., Brandner, W., Lehmann, T., Weigelt, G., \& Zinnecker, H. 1996, A\&A, 315, 445

Simon, M., Ghez, A. M., Leinert, C., et al. 1995, ApJ, 443, 625

Shakura, N. I., \& Sunyaev, R. A. 1973, A\&A, 24, 337

Skinner, S. L., Brown, A., \& Steart, R.T. 1993, ApJS, 87, 217

Strom, K. M., \& Strom, S. E. 1994, ApJ, 424, 237

Tout, C. A., \& Pringle, J. P. 1995, MNRAS, 272, 528

Weigelt, G. 1977, Opt. Commun., 21, 55

Weigelt, G., \& Wirnitzer, B. 1983, Opt. Lett., 8, 389

Yudin, R. V. 2000, A\&AS, 144, 285

Zinnecker, H., \& Preibisch, T. 1994, A\&A, 292, 152

Zinnecker, H., \& Preibisch, T. 1995, Ap\&SS, 224, 587 